\title{
Intergrain Effects in the AC Susceptibility of Polycrystalline $\mathrm{LaFeAsO}_{0.94} \mathrm{~F}_{0.06}$
}

\author{
G. Bonsignore • A. Agliolo Gallitto • M. Li Vigni • \\ J.L. Luo • G.F. Chen · N.L. Wang • D.V. Shovkun
}

Received: 20 July 2010 / Accepted: 2 September 2010 / Published online: 18 September 2010

(C) Springer Science+Business Media, LLC 2010

\begin{abstract}
The AC susceptibility, $\chi$, at zero DC magnetic field of a polycrystalline sample of $\mathrm{LaFeAsO}_{0.94} \mathrm{~F}_{0.06}\left(T_{c} \approx 24 \mathrm{~K}\right)$ has been investigated as a function of the temperature, the amplitude of the AC magnetic field (in the range $H_{a c}=0.003 \mathrm{Oe} \div$ $4 \mathrm{Oe}$ ) and the frequency (in the range $f=10 \mathrm{kHz} \div 100 \mathrm{kHz}$ ). The $\chi(T)$ curve exhibits the typical two-step transition arising from the combined response of superconducting grains and intergranular weak-coupled medium. The intergranular part of $\chi$ strongly depends on both the amplitude and the frequency of the AC driving field, from few Kelvin below $T_{c}$ down to $T=4.2 \mathrm{~K}$. Our results show that, in the investigated sample, the intergrain critical current is not determined by pinning of Josephson vortices but by Josephson critical current across neighboring grains.
\end{abstract}

Keywords Oxypnictides · AC susceptibility · Granularity $\cdot$ Josephson junction array

\section{Introduction}

The discovery [1] of superconductivity in iron-based oxypnictides, $\mathrm{ReFeAsO}_{1-x} \mathrm{~F}_{x}$ with Re: rare earth, has attracted much attention by the condensed-matter-physics

G. Bonsignore · A. Agliolo Gallitto · M. Li Vigni $(\varangle)$

CNISM and Dipartimento di Scienze Fisiche ed Astronomiche, Università di Palermo,

Via Archirafi 36, 90123 Palermo, Italy

e-mail: livigni@fisica.unipa.it

J.L. Luo · G.F. Chen · N.L. Wang

Beijing National Laboratory for Condensed Matter Physics, Institute of Physics, Chinese Academy of Sciences, Beijing 100190, China

D.V. Shovkun

Institute of Solid State Physics, Russian Academy of Sciences, Chernogolovka, 142432 Moscow, Russia 
community. Such compounds exhibit magnetic order and superconductivity on varying the doping; furthermore, the critical temperature strongly depends on the fluorine content [2]. In optimally doped samples, $T_{c}$ varies in the range $20 \mathrm{~K} \div 50 \mathrm{~K}$ depending on the $\mathrm{Re}$ ion. The main reason of this interest is the similarity between some properties of iron-based pnictides and those of cuprate superconductors, such as the layered structure, low carrier density, magnetic order in parent (undoped) compounds, possible unconventional pairing mechanism. Moreover, several studies have highlighted that the superconducting-gap structure of iron-based pnictides resembles that of $\mathrm{MgB}_{2}$, with multi-gap superconductivity [3-6], which could originate from their multiple-electronic-band structures.

One of the characteristics of high- $T_{c}$ cuprates is related to their granularity [7]; due to the small value of the coherence length, grain boundaries as well as defects even of small dimensions act as weak links, giving rise to flux penetration at low magnetic fields [8], depression of the critical current density, nonlinear effects [9, 10]. On the contrary, it has been extensively shown that in $\mathrm{MgB}_{2}$ only a small number of grain boundaries act as weak links [11], giving rise to reduced nonlinear effects [12]. In this scenario, it is of importance to explore granularity effects in iron-based oxypnictides and compare the results with those obtained in other superconductors.

Investigation of the electromagnetic response of different polycrystalline samples of iron-based oxypnictides have highlighted granularity effects, such as two-step susceptibility transition [13]; different values of the global current density, flowing through weak-coupled grains, and the local current density, inside the superconducting grains [14]; small hysteresis in DC magnetization of bulk and powdered samples [15]. However, it has not been clarified if such effects are due to intrinsic properties of the oxypnictides or are related to specific properties of the investigated samples.

Measurements of the AC susceptibility can be conveniently used to investigate both intergrain and intragrain flux dynamics in superconductors [7, 16-22]. In particular, measurements at low DC magnetic fields as a function of the AC-field amplitude allows to highlight possible nonlinear effects related to critical state in the intergrain region; investigation as a function of the driving-field frequency allows to identify the different regimes of flux motion.

In this paper, we investigate the $\mathrm{AC}$ susceptibility of a sample of $\mathrm{LaFeAsO}_{1-x} \mathrm{~F}_{x}$, with $x=0.06$, and compare the results with those obtained in ceramic cuprates. The measurements have been performed at zero DC magnetic field as a function of the temperature, for different values of the amplitude and the frequency of the driving field, and at $T=4.2 \mathrm{~K}$ as a function of the AC-field amplitude. The results are qualitatively discussed in the framework of the models reported in the literature for the electrodynamic response of the intergrain region. A qualitative comparison of the experimental $\chi\left(H_{a c}, f\right)$ curves with those obtained by Chen et al. [22] in the framework of the so-called flux-flow critical-state model has highlighted that, in the investigated sample, the intergrain critical current is not determined by pinning of Josephson vortices but by Josephson critical current across neighboring grains. 


\section{Experimental apparatus and sample}

The AC susceptibility, $\chi=\chi^{\prime}+i \chi^{\prime \prime}$, has been measured in a polycrystalline sample of $\mathrm{LaFeAsO}_{0.94} \mathrm{~F}_{0.06}$, prepared by solid state reaction using $\mathrm{LaAs}, \mathrm{Fe}_{2} \mathrm{O}_{3}, \mathrm{Fe}$ and $\mathrm{LaF}_{3}$ as starting materials. The sample has a nearly parallelepiped shape of approximate dimensions $1 \times 2 \times 2 \mathrm{~mm}^{3}$; details on the preparation and properties of the sample are reported in Ref. [23]. Atomic-Force-Microscopy (AFM) analysis on the sample surface has highlighted a high inhomogeneity; in particular, the sample seems to consist of grains of micrometric size embedded in a finer grained matrix.

The measurements have been performed by a computer controlled mutualinductance susceptometer, which uses two identical secondary coils oppositely wounded. The coils are positioned coaxially inside a long primary coil, which provides an uniform $\mathrm{AC}$ field at the specimen position. The sample is located at the center of one of the secondary coils, by a sapphire holder, with the largest surface perpendicular to the coil axis. A temperature sensor and a heater are also fixed at the sapphire holder. The induced disbalance voltage in the secondary coils is proportional to the sample susceptibility: $v=\alpha f H_{a c} \chi$, where $\alpha$ depends on the sample and coil geometry. The signal is detected by a lock-in amplifier that allows to separate the real, $\chi^{\prime}$, and the imaginary, $\chi^{\prime \prime}$, components. To reduce the change of the background disbalance signal, the coils are maintained in a liquid He bath, while sample, sensor and heater are placed in gaseous He.

The AC susceptibility in the absence of DC magnetic field has been investigated as a function of the temperature, the amplitude of the $\mathrm{AC}$ driving field (in the range $H_{a c} \approx 0.003 \mathrm{Oe} \div 4 \mathrm{Oe}$ ) and the frequency (in the range $f=10 \mathrm{kHz} \div 100 \mathrm{kHz}$ ). In order to disregard the constant $\alpha$, we have measured the $\chi^{\prime}$ vs $H_{a c}$ curve at $T=4.2 \mathrm{~K}$; extrapolating the curve to $H_{a c} \rightarrow 0$, we have determined a constant, $\chi_{0}$, which has been used to normalize all the curves here reported.

\section{Experimental results}

Figure 1 shows the normalized value of $\chi^{\prime}$ and $\chi^{\prime \prime}$ as a function of the temperature, obtained at zero DC field and $H_{a c}=0.007 \mathrm{Oe}$, at different values of the frequency (from $10 \mathrm{kHz}$ to $100 \mathrm{kHz}$ ). The $\chi^{\prime}(T)$ curves show a two-step transition typical of granular superconductors $[16,17]$. In correspondence of the low- $T$ step, the $\chi^{\prime \prime}(T)$ curve shows a well-defined peak, at a characteristic temperature $T_{p}$. On increasing the frequency, the peak intensity increases and $T_{p}$ moves towards higher temperatures. The inset shows the dependence of $T_{p}$ on the driving-field frequency. On the contrary, in correspondence of the near- $T_{c}$ step we observe only a "kink", better visible in the figure at high frequencies.

The frequency dependence of $\chi$ reported in Fig. 1 is much more enhanced than that reported in the literature for high- $T_{c}$ cuprates [18-20, 24]. For example, Nikolo and Goldfarb [18], investigating the frequency dependence of AC susceptibility in ceramic YBCO, observed a shift of $T_{p}$ with the frequency, from $10 \mathrm{~Hz}$ to $1000 \mathrm{~Hz}$, of few tenths of $\mathrm{K}$ at small $H_{a c}$ and of the order of $1 \mathrm{~K}$ at $H_{a c}=10 \mathrm{Oe}$. On the contrary, at $H_{a c}=0.007 \mathrm{Oe}$, varying the frequency from $10 \mathrm{kHz}$ to $100 \mathrm{kHz}$ we observe a $T_{p}$ shift of about $4 \mathrm{~K}$. 


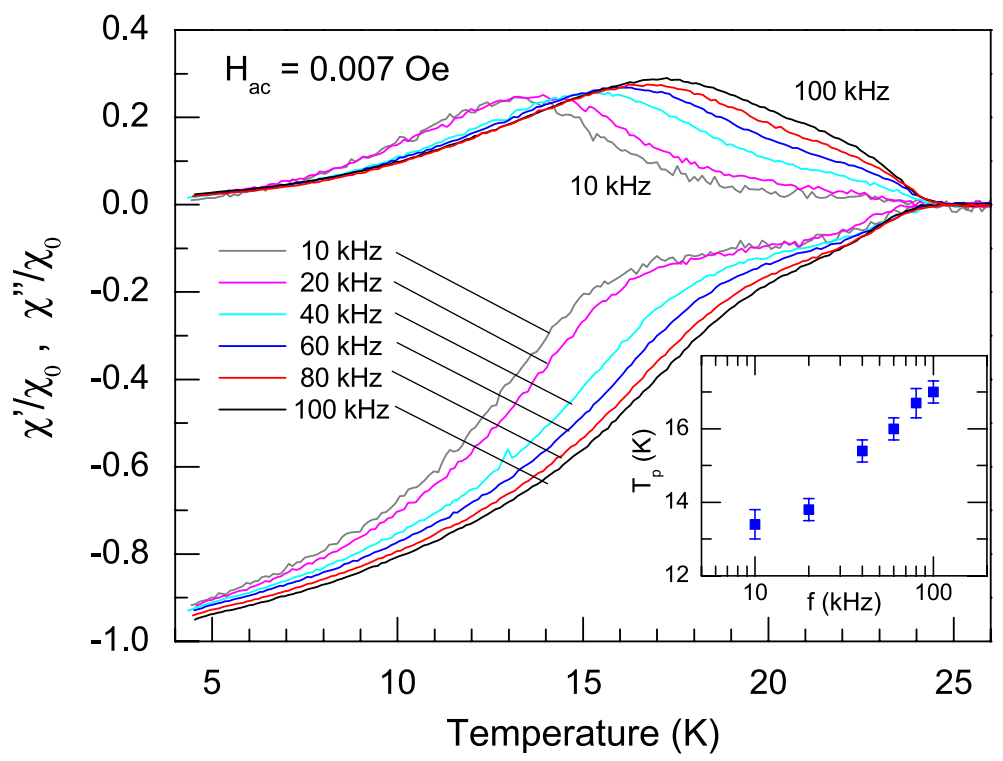

Fig. 1 (Color online) Normalized values of $\chi^{\prime}$ and $\chi^{\prime \prime}$ as a function of the temperature, obtained at $H_{a c}=0.007$ Oe and different values of the frequency. Inset: dependence of $T_{p}$ on the driving-field frequency

Figure 2 shows the temperature dependence of $\chi^{\prime}$ and $\chi^{\prime \prime}$ at the two frequencies $f=100 \mathrm{kHz}$ and $f=10 \mathrm{kHz}$, obtained at zero DC magnetic field and different values of the AC-field amplitude. One can note a near- $T_{c}$ region characterized by a linear response (independent of $H_{a c}$ ) and a region at lower $T$ highlighting enhanced nonlinear effects.

In Fig. 3, we report the dependence of $\chi^{\prime}$ and $\chi^{\prime \prime}$ on the amplitude of the AC field, obtained at $T=4.2 \mathrm{~K}$ and at the two frequencies: $f=100 \mathrm{kHz}$ and $f=10 \mathrm{kHz}$. Even at low temperatures, the susceptibility strongly depends on the frequency; in particular, the AC-field value at which we observe a maximum in $\chi^{\prime \prime}\left(H_{a c}\right)$ increases of a factor of four on varying the frequency from $10 \mathrm{kHz}$ to $100 \mathrm{kHz}$, and the maximum value of $\chi^{\prime \prime} / \chi_{0}$ varies from $\approx 0.21$ to $\approx 0.27$.

By analyzing the results of Figs. 2 and 3 and other data not reported here, we have obtained the dependence of $T_{p}$ on the amplitude of the AC field; the results are shown in Fig. 4. One can note a strong field dependence even at low $H_{a c}$ values; it is worth to remark that in high- $T_{c}$ cuprates $T_{p}$ nearly saturates for $H_{a c} \lesssim 0.1$ Oe $[19,24]$.

\section{Discussion}

Investigation carried out since the discovery of cuprate superconductors has shown that the AC susceptibility transition, with two steps in $\chi^{\prime}(T)$ accompanied by two peaks in $\chi^{\prime \prime}(T)$, is expected in samples consisting of superconducting grains interconnected by a system of weak links $[16,17]$. When cooled below $T_{c}$, the grains first become superconducting, and shielding is performed by the so-called intragrain 


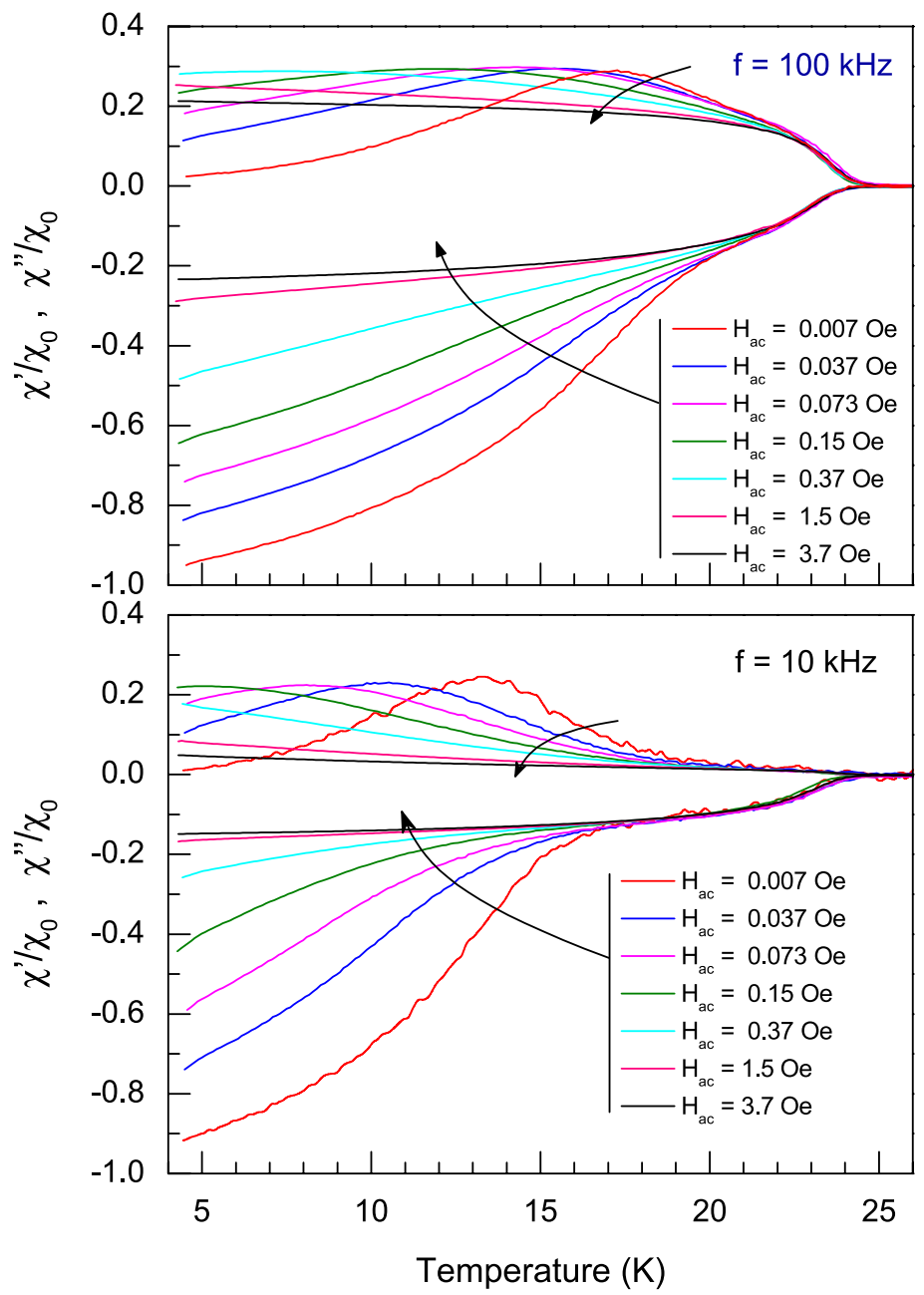

Fig. 2 (Color online) Real and imaginary components of the AC susceptibility as a function of the temperature, obtained at zero DC field and different value of $H_{a c}$, for $f=100 \mathrm{kHz}$ and $f=10 \mathrm{kHz}$. Arrows indicate increasing $H_{a c}$ values

current, with density $J_{c g}$. On further decreasing the temperature, the whole sample behaves as a homogeneous superconductor able to carry the macroscopic intergrain current density $J_{c m}$, which is determined by the Josephson current across neighboring grains. In this case, one expects to detect in $\chi^{\prime \prime}(T)$ a near- $T_{c}$ peak, which occurs when the AC field penetrates just to the center of grains, and a lower- $T$ peak corresponding to complete penetration of the AC field in the weak-link matrix. It is worth to remark that a near- $T_{c}$ peak, indicated as intrinsic peak, would occur even in the case of reversible screening (negligible intragrain pinning); it is expected at the temperature at which the AC-field penetration depth becomes comparable with the grain size. 
Fig. 3 (Color online) $\chi^{\prime}$ and $\chi^{\prime \prime}$ as a function of the amplitude of the AC magnetic field, at two different values of the frequency

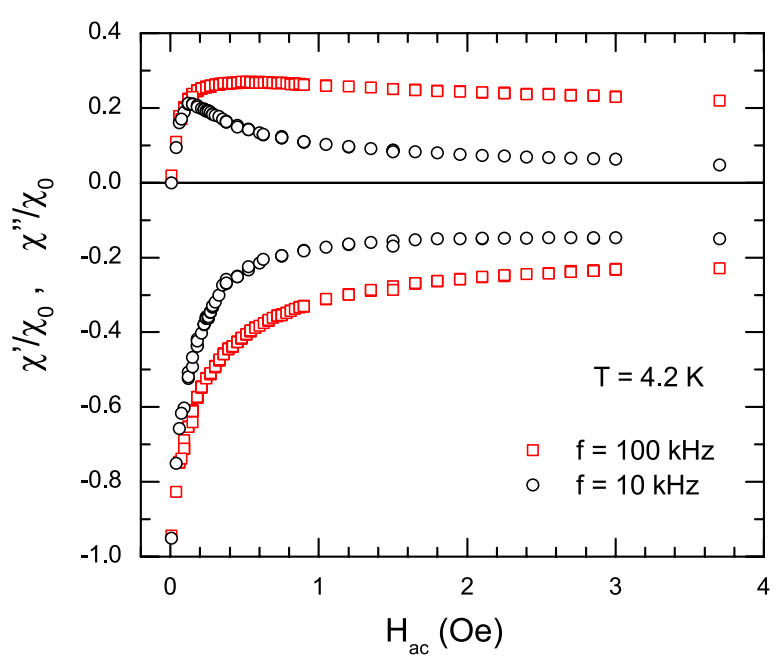

Fig. 4 (Color online)

Temperature position of the $\chi^{\prime \prime}$ peak as a function of the amplitude of the AC magnetic field, at two different values of the frequency. The points with horizontal error bars have been deduced from the data of Fig. 3

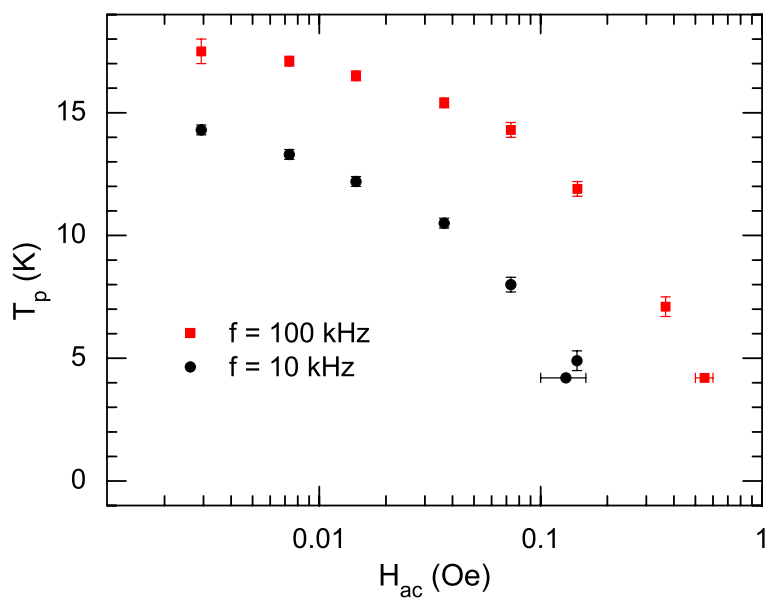

From Fig. 1, one can easily distinguish two steps in $\chi^{\prime}(T)$; according to the above considerations, the step near $T_{c}$ is ascribable to the superconducting-grain shielding, while that at lower $T$ to the intergrain shielding. In correspondence of the low- $T$ step, we observe a well defined peak in $\chi^{\prime \prime}(T)$, while the expected near- $T_{c}$ peak is not well resolved. We observe only a kink at $T \approx 22 \mathrm{~K}$, better visible at high frequency; this finding could be due to a wide grain-size distribution and/or a wide distribution of $J_{c g}$. On the other hand, the inhomogeneity of grains does not hinder shielding inside each grain, whose effect is well visible in $\chi^{\prime}(T)$.

In order to understand if the near- $T_{c}$ response of grains is related to reversible or irreversible screening, it is convenient to look at the $\chi$ vs $T$ curves for different $H_{a c}$. From Fig. 2, one can see that for $T \gtrsim 20 \mathrm{~K}$, the $\chi$ vs $T$ curves do not depend on $H_{a c}$ (linear response), while at lower $T$ enhanced nonlinear response is observed. Irreversible response during the $\mathrm{AC}$-field cycle gives rise to nonlinear effects, which 
manifest themselves with components of $\chi$ oscillating at harmonic frequencies of the driving field as well as with dependence of $\chi^{\prime}$ and $\chi^{\prime \prime}$ on $H_{a c}[9,10,17]$. The results we obtained near $T_{c}$ strongly suggest that the intragrain response is related to reversible screening, because of weak intragrain pinning at $T \gtrsim 20 \mathrm{~K}$.

According to Chen et al. [25], the measured sample susceptibility can be written as the sum of the intergranular susceptibility, $\chi_{m}$, and the grain susceptibility, $\chi_{g}$, weighted by the effective volume fractions of the intergranular region and superconducting grains (excluding a shell of thickness of the order of the field penetration depth).

At $T \ll T_{c}$ and for magnetic fields smaller than the lower critical field of grains it is expected that $\chi_{g}^{\prime}=-1$ and $\chi_{g}^{\prime \prime}=0$; so, the sample susceptibility results

$$
\begin{aligned}
\chi^{\prime} & =-f_{g}+\left(1-f_{g}\right) \chi_{m}^{\prime}, \\
\chi^{\prime \prime} & =\left(1-f_{g}\right) \chi_{m}^{\prime \prime},
\end{aligned}
$$

where $f_{g}$ is the volume fraction of the grains and $1-f_{g}$ that of the intergrain region.

Looking at Figs. 2 and 3, the variations of $\chi$ at low temperatures are ascribable to the intergrain contribution. On increasing $H_{a c}$, the field penetrates the intergrain region reducing the shielding until $\chi^{\prime}$ reaches a saturation value ascribable to the grain shielding. Correspondingly, $\chi^{\prime \prime}$ increases, reaches the maximum value when the field penetrates, through the intergrain medium, to the center of the sample, and then decreases. The results we obtained for $\chi^{\prime}$ allows to affirm that in the investigated sample the volume fraction of the superconducting grains able to shield is less than the $20 \%$ of the whole sample volume; the presence of grains as small as the penetration depth reduces the shielding but contributes to the transport of the intergrain current.

Analysis of the results in the nonlinear regime can give useful information on the processes occurring in the intergrain region. In the framework of the Bean criticalstate (CS) model, the value of $H_{a c}$ at which the $\chi^{\prime \prime}$ peak occurs roughly coincides with the full penetration field of the intergrain region, $H_{m}^{*}$. From Fig. 3 at $T=4.2 \mathrm{~K}$, we obtain $H_{m}^{*} \approx 0.55 \mathrm{Oe}$ at $f=100 \mathrm{kHz}$ and $H_{m}^{*} \approx 0.13$ Oe at $f=10 \mathrm{kHz}$. These values are very small and should indicate low values of the intergrain critical current density or intergrain-current path around regions much smaller than the sample dimension. In order to check if the latter hypothesis applies, we have coarsely crushed a portion of the sample, obtaining a powder with fragments of dimensions ranging from $50 \mu \mathrm{m}$ to $500 \mu \mathrm{m}$. Figure 5 shows $\chi^{\prime \prime}$ as a function of the AC-field amplitude in the pristine and crushed samples, at $T=4.2 \mathrm{~K}$ and $f=100 \mathrm{kHz}$. One can see that $H_{m}^{*}$ in the crushed sample is about one order of magnitude smaller than that obtained in the pristine sample. Since the ratio of the two $H_{m}^{*}$ is of the same order of that between the dimension of the bulk sample and the mean size of the powder grains, we can rule out the possibility to have intergrain-current path much smaller than the whole bulk sample (i.e. the weak-link matrix extents over the whole sample).

It is worth noting that we observe a strong frequency dependence of both the height and the position of the $\chi^{\prime \prime}$ peak, which is not expected in the mere CS. Frequency dependence of the AC susceptibility has been discussed by different authors in the framework of different models [19, 21, 22, 26, 27]. Muller [19] considers the granular superconductor as a regular array of Josephson junctions connecting cubic grains, 
Fig. 5 (Color online) Comparison between the $H_{a c}$ dependence of $\chi^{\prime \prime}$, obtained in the bulk and crushed samples

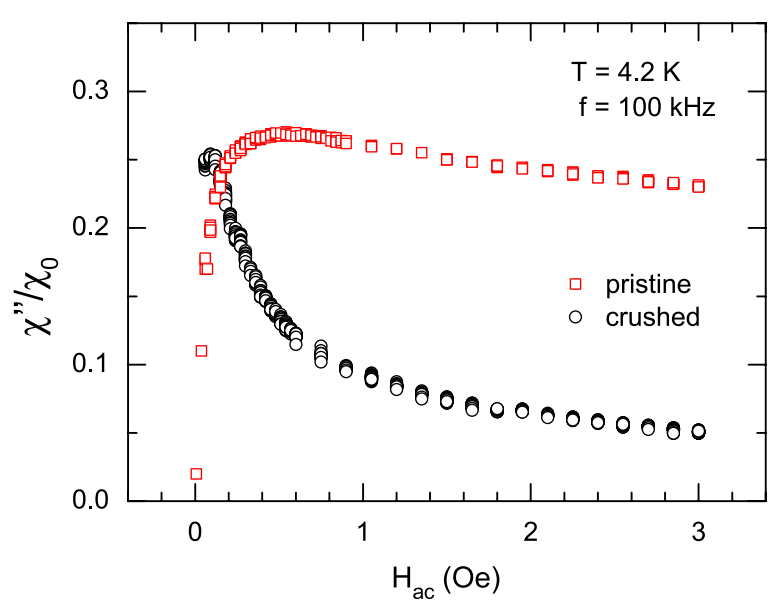

and Josephson vortices are assumed to hop between adjacent pinning centers located at the grain corners. In the framework of this model, the frequency dependence is ascribed to effects of magnetic relaxation during the $\mathrm{AC}$ cycle and it is taken into account by adding a flux-creep term to the current term in the CS equation. Other authors $[26,27]$ take into account the magnetic relaxation by assuming a proper frequency dependence of the intergranular critical current.

Polichetti et al. [21] have solved the nonlinear equation describing the diffusion of the magnetic field inside the sample, hypothesizing a proper dependence of the pinning potential on the current density, $U(J)$. Numerical computation of AC susceptibility using different $U(J)$ laws, corresponding to the linear dependence in the Kim-Anderson model and the nonlinear ones in the vortex-glass models, has highlighted a way to distinguish the different vortex phases. In particular, on increasing the frequency the height of the loss peak in the $\chi^{\prime \prime}(T)$ curve is expected to increase in the vortex-glass phase and to decrease in the Kim-Anderson case [21]. From Fig. 1, one can see that on increasing the frequency the height of the $\chi^{\prime \prime}(T)$ peak increases, suggesting that the intergrain-vortex lattice could be in the vortex-glass phase.

Although the above-mentioned models predict a $\chi^{\prime \prime}(T)$-peak shift towards higher temperatures with the frequency, the shift we observe is much more enhanced than the expected one, no matter the creep exponent used. Moreover, we observe a strong dependence of the susceptibility on both the amplitude and frequency of the AC field even at low temperatures (see Fig. 3) where thermal activated flux-creep should be slightly effective. So, our results cannot fully be explained by considering flux-creep effects.

Chen et al. [28] have investigated the AC susceptibility of a sintered YBCO superconductor as a function of the amplitude and frequency of the driving field at fixed temperatures. In order to recognize the mechanisms determining the intergranular critical current at the different frequencies, they have numerically calculated $\chi\left(H_{a c}, f\right)$ using different $E(J)$ characteristics, in the different motion regimes (collective flux creep, Kim-Anderson flux creep and flux flow). By using, for the flux-flow case, $E(J)=\left[J-\operatorname{sgn}(J) J_{c}\right] \rho_{f}$ occurring at $|J|>J_{c}$ with $\rho_{f}$ the flux-flow resistivity, the authors show that the experimental results are consistent with those obtained 
Fig. 6 (Color online) Symbols: real and imaginary components of the intergranular $\mathrm{AC}$ susceptibility of the $\mathrm{LaFeAsO}_{0.94} \mathrm{~F}_{0.06}$ sample, $\chi_{m}^{\prime}$ and $\chi_{m}^{\prime \prime}$, as a function of the amplitude of the AC magnetic field, obtained at $T=4.2 \mathrm{~K}$ for $f=10 \mathrm{kHz}$ and $f=100 \mathrm{kHz}$; the results have been obtained from the data of Fig. 3 supposing the volume fraction of grains to be $f_{g}=0.14$. Continuous lines show the results expected in the Bean CS model with $J_{c}=1 \times 10^{4} \mathrm{~A} / \mathrm{m}^{2}$

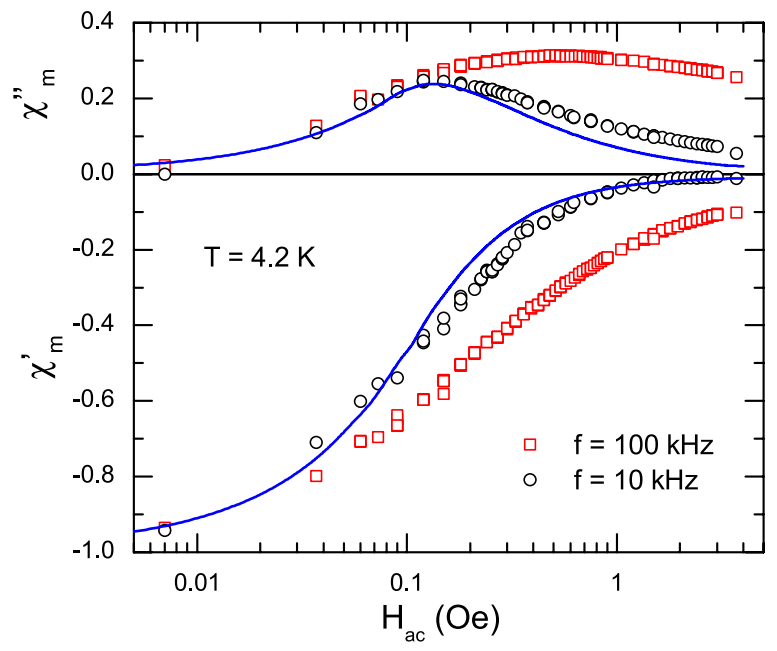

by the so-called flux-flow critical-state (FFCS) model [22]. In this model, $J_{c m}$ arises directly from the Josephson currents across weak-linked grains and the flux-flow behavior should be a consequence of the resistively shunted Josephson-junction network.

The $\chi\left(H_{a c}, f\right)$ curves obtained by the FFCS model have different features with respect to those obtained from the flux-creep models. In particular: i) the region of the curves for $H_{a c}$ values smaller than the value at which $\chi^{\prime \prime}$ exhibits the maximum are slightly affected by the frequency variations; ii) on increasing the frequency, the maximum of the $\chi^{\prime \prime}\left(H_{a c}\right)$ curve shifts towards higher $H_{a c}$, its value increases and the peak broadens; iii) on increasing the frequency, $\chi^{\prime}\left(H_{a c}\right)$ grows more slowly with $H_{a c}$, reaching its saturation value at higher $H_{a c}$. In Fig. 6, we report as symbols the susceptibility of the $\mathrm{LaFeAsO}_{0.94} \mathrm{~F}_{0.06}$ sample as a function of $\log \left(H_{a c}\right)$, properly normalized to extract $\chi_{m}$; in particular, we have set the volume fraction of the grains $f_{g}=0.14$. Comparing these curves with those reported in Fig. 2 of Ref. [28], one can note that $\chi_{m}\left(H_{a c}, f\right)$ curves we obtained are consistent with the results expected from the FFCS model.

An important result highlighted by Chen et al. is that the intergrain susceptibility calculated by the FFCS model in the low-frequency limit coincides with that obtained by using the Bean CS model. Since the experimental $\chi^{\prime \prime}\left(H_{a c}\right)$-peak value at $10 \mathrm{kHz}$ is compatible with that expected in the Bean CS model, we have calculated the $\chi_{m}\left(H_{a c}\right)$ curves by using the Bean CS model. In order to take into account the demagnetization effects, we have set

$$
H_{a c}^{e f f}=H_{a c}-N M_{m}-n M_{g},
$$

where $N$ is the demagnetization factor related to the sample shape and $M_{m}$ the intergrain magnetization; $n$ and $M_{g}$ are the demagnetization factor and the magnetization of grains, respectively. Supposing grains have spherical shape and completely screen the field, we have set $n=1 / 3$ and $M_{g}=-f_{g} H_{a c}^{e f f}$. 
In order to determine $\chi_{m}$, we have calculated the sample magnetization considering a straight-line flux-density profile of slope $J_{c}$ during the AC-field cycle. The contribution to $H_{a c}^{e f f}$ of the intergrain matrix has been taken into account by the following iterative procedure. In the first step, we have calculated $M_{m}$ setting $H_{a c}^{e f f}=H_{a c}$, with this value of $M_{m}$ and using (1) we obtain a new $H_{a c}^{e f f}$ value; the procedure is repeated until two consecutive values of $M_{m}$ differ by less than $1 \%$. The converging value of $M_{m}$ has been used to calculate $\chi_{m}$ by

$$
\chi_{m}=\frac{1}{\pi H_{a c}} \int_{0}^{2 \pi} M_{m}(t) e^{i \omega t} \mathrm{~d}(\omega t) .
$$

The $\chi_{m}$ vs $H_{a c}$ curves calculated by this procedure are shown in Fig. 6 as continuous lines; they have been obtained with $J_{c}=1 \times 10^{4} \mathrm{~A} / \mathrm{m}^{2}$ independent of $H_{a c}$. As one can see, the calculated $\chi_{m}\left(H_{a c}\right)$ curves account quite well for the experimental ones up to $H_{a c} \approx 0.2 \mathrm{Oe}$. The small discrepancy at higher AC fields between the experimental curves obtained at $10 \mathrm{kHz}$ and the calculated ones may be due to the fact that the low-frequency limit is not fully attained in the sample. We have also calculated $\chi_{m}$ supposing an exponential field dependence of $J_{c}$, but it gave a worse fit. The experimental curves at $100 \mathrm{kHz}$ are consistent with those obtained by Chen et al. [22, 28 ] by the FFCS model in the eddy-current limit, with a broad and more intense $\chi_{m}^{\prime \prime}\left(H_{a c}\right)$ peak and lower values of $\chi^{\prime}\left(H_{a c}\right)$ with respect to the curves at $10 \mathrm{kHz}$.

The consistency of our experimental results with the theoretical ones obtained by Chen et al. in the framework of the FFCS model suggests that in the investigated sample the intergrain critical current is not determined by pinning of Josephson vortices but by the Josephson critical current across grain boundaries. This means that the intergrain region cannot be described as a type-II superconductor in the critical state, whose electrodynamics is governed by the fluxon pinning, but a better representation can be done considering it as a network of resistively shunted Josephson junctions. It would be interesting to investigate samples of $\mathrm{LaFeAsO}_{1-\delta} \mathrm{F}_{\delta}$ with different fluorine content, and/or prepared by different methods, in order to understand if the intergrain properties are related to specific properties of the investigated sample or to intrinsic properties of iron-based oxypnictides.

\section{Conclusion}

We have investigated the AC susceptibility of a sample of $\mathrm{LaFeAsO}_{0.94} \mathrm{~F}_{0.06}$, in the absence of static magnetic field, in the range of frequency $f=10 \mathrm{kHz} \div 100$ $\mathrm{kHz}$. The real and imaginary components of $\chi$ have been measured as a function of the temperature, at different values of the amplitude and the frequency of the AC field, and at $T=4.2 \mathrm{~K}$ as function of the AC-field amplitude, at the two frequencies $f=10 \mathrm{kHz}$ and $f=100 \mathrm{kHz}$.

The $\chi(T)$ curves exhibit a two-step transition due to the intergranular and intragranular response. Measurements at different $H_{a c}$ have highlighted a linear response at $T \gtrsim 20 \mathrm{~K}$, ascribable to reversible screening of the superconducting grains. The results obtained for lower temperatures have highlighted that the intergrain susceptibility strongly depends on the amplitude and frequency of the AC field. On increasing 
$H_{a c}$ the intergrain shielding reduces and approaches its saturation value, corresponding to the complete shielding of grains, at applied field of the order of few Oe. This allowed us to estimate the volume fraction of the superconducting grains able to shield the field to be roughly of $15 \%$ of the whole sample volume. This finding can be ascribed to the presence in the sample of very small grains, as highlighted by AFM measurements.

Analysis of the results at low temperatures have been qualitatively discussed in the framework of models reported in the literature for the electrodynamic response of the intergrain medium. The features of the intergranular $\chi\left(H_{a c}, f\right)$ curves are consistent with the results obtained in the framework of the so-called flux-flow critical-state model, developed by Chen et al. for describing the electromagnetic response of the intergrain medium. In particular, we have shown that the $\chi\left(H_{a c}\right)$ curves obtained at $10 \mathrm{kHz}$ are in qualitative agreement with the results expected from the Bean criticalstate model, consistently with the results obtained by Chen et al. in the low-frequency limit; the experimental results obtained at $100 \mathrm{kHz}$ are consistent with those obtained, by the same model, in the flux-flow regime. This finding strongly suggests that in the investigated $\mathrm{LaFeAsO}_{0.94} \mathrm{~F}_{0.06}$ sample the intergrain region can be considered as a resistively shunted Josephson-junction array, in which the critical current is determined by the maximum Josephson current across neighboring grains but not by pinning of Josephson vortices.

Acknowledgements The authors are very glad to thank Massimiliano Polichetti and Marco Bonura for the interest on this work and fruitful suggestions. Work partially supported by the University of Palermo in the framework of the International Co-operation Project CoRI 2007 Cupane.

\section{References}

1. Y. Kamihara, T. Watanabe, M. Hirano, H. Hosono, J. Am. Chem. Soc. 130, 3296 (2008)

2. G.F. Chen, Z. Li, D. Wu, J. Dong, G. Li, W.Z. Hu, P. Zheng, J.L. Luo, N.L. Wang, Phys. Rev. Lett. 101, 57007 (2008)

3. S. Kawasaki, K. Shimada, G.F. Chen, J.L. Luo, N.L. Wang, G.-Q. Zheng, Phys. Rev. B 78, 220506(R) (2008)

4. P. Samuely, P. Szabó, Z. Pribulová, M.E. Tillman, S. Bud'ko, P.C. Canfield, Supercond. Sci. Technol. 22, 014003 (2009)

5. R.S. Gonnelli, D. Daghero, M. Tortello, G.A. Ummarino, V.A. Stepanov, J.S. Kim, R.K. Kremer, Phys. Rev. B 79, 184526 (2009)

6. G.A. Ummarino, M. Tortello, D. Daghero, R.S. Gonnelli, Phys. Rev. B 80, 172503 (2009)

7. J.R. Clem, Physica C 153-155, 50 (1988)

8. Y. Kim, G.H. Lam, C.D. Jeffries, Phys. Rev. B 43, 11404 (1991)

9. M.G. Adesso, C. Senatore, M. Polichetti, S. Pace, Physica C 404, 289 (2004)

10. I. Ciccarello, M. Guccione, M. Li Vigni, Physica C 161, 39 (1989)

11. S.B. Samanta, H. Narayan, A. Gupta, A.V. Narlikar, T. Muranaka, J. Akimitsu, Phys. Rev. B 65, 092510 (2002)

12. A. Agliolo Gallitto, G. Bonsignore, E. Di Gennaro, G. Giunchi, M. Li Vigni, P. Manfrinetti, Microw. Opt. Technol. Lett. 48, 2482 (2006)

13. M. Polichetti, M.G. Adesso, D. Zola, J.L. Luo, G.F. Chen, Z. Li, N.L. Wang, C. Noce, S. Pace, Phys. Rev. B 78, 224523 (2008)

14. A. Yamamoto, A.A. Polyanskii, J. Jiang, F. Kametani, C. Tarantini, F. Hunte, J. Jaroszynski, E.E. Hellstrom, P.J. Lee, A. Gurevich, D.C. Larbalestier, Z.A. Ren, J. Yang, X.L. Dong, W. Lu, Z.X. Zhao, Supercond. Sci. Technol. 21, 095008 (2008) 
15. A. Yamamoto, J. Jiang, C. Tarantini, N. Craig, A.A. Polyanskii, F. Kametani, F. Hunte, J. Jaroszynski, E.E. Hellstrom, D.C. Larbalestier, R. Jin, A.S. Sefat, M.A. McGuire, B.C. Sales, D.K. Christen, D. Mandrus, Appl. Phys. Lett. 92, 252501 (2008)

16. F. Gömöry, Supercond. Sci. Technol. 10, 523 (1997) and references therein

17. R.A. Hein, T.L. Francavilla, D.H. Liebenberg (eds.), Magnetic Susceptibility of Superconductors and Other Spin Systems (Plenum Press, New York, 1991)

18. M. Nikolo, R.B. Goldfarb, Phys. Rev. B 39, 6615 (1989)

19. K.H. Muller, Physica C 168, 585 (1990)

20. I. Dhingra, B.K. Das, Supercond. Sci. Technol. 6, 765 (1993)

21. M. Polichetti, M. Adesso, S. Pace, Physica A 339, 119 (2004)

22. D.-X. Chen, E. Pardo, A. Sanchez, Appl. Phys. Lett. 86, 242503 (2005)

23. G.F. Chen, Z. Li, G. Li, J. Zhou, D. Wu, J. Dong, W.Z. Hu, P. Zheng, Z.J. Chen, J.L. Luo, N.L. Wang, Phys. Rev. Lett. 101, 57007 (2008)

24. N. Savvides, A. Katsaros, C. Andrikidis, K.H. Muller, Physica C 197, 267 (1992)

25. D.-X. Chen, J. Nogues, K.V. Rao, Cryogenics 29, 800 (1989)

26. B.J. Jonsson, K.V. Rao, S.H. Yun, U.O. Karlsson, Phys. Rev. B 58, 5862 (1998)

27. O. Ozogul, Phys. Status Solidi 202, 1793 (2005)

28. D.-X. Chen, E. Pardo, A. Sanchez, E. Bartolomé, Appl. Phys. Lett. 89, 072501 (2006) 